\title{
Greater Medial Meniscus Extrusion Seen on Ultrasonography Indicates the Risk of Posterior Root Tear in Non-radiographic Knee Osteoarthritis Population
}

\section{Daisuke Chiba ( $\nabla$ dachiba@hirosaki-u.ac.jp)}

Department of Orthopaedic Surgery, Hirosaki Memorial Hospital

\section{Tomoyuki Sasaki}

Department of Orthopaedic Surgery, Hirosaki Memorial Hospital

Yasuyuki Ishibashi

Department of Orthopaedic Surgery, Hirosaki University Graduate School of Medicine

\section{Research Article}

Keywords: Ultrasound, Magnetic resonance imaging, Medial meniscus extrusion, Posterior root tear

Posted Date: July 22nd, 2021

DOl: https://doi.org/10.21203/rs.3.rs-729085/v1

License: (c) (1) This work is licensed under a Creative Commons Attribution 4.0 International License.

Read Full License 


\section{Abstract}

Purpose: To elucidate the association between medial meniscus extrusion measured on ultrasonography $\left(\mathrm{MME}_{\mathrm{US}}\right)$ and the prevalence of medial meniscus posterior root tear detected on magnetic resonance imaging (MMPRT ${ }_{\text {MRI }}$ ).

Methods: We recruited 127 patients (135 knees) in this cross-sectional study. All participants had medial knee pain without a knee trauma or surgery history. Knee osteoarthritis (KOA) severity was evaluated using Kellgren-Lawrence grade (KLG) scores. Patients with KLG scores 0-1 and $\geq 2$ were classified in nonradiographic (non-ROA) and radiographic KOA (ROA) groups, respectively. $\mathrm{MME}_{\mathrm{US}}$ was measured with patients in the supine position. Based on T2*-weighted images, MMPRT ${ }_{M R I}$ was defined as the presence of "Ghost meniscus sign" and "Creft/truncation sign", indicating an abnormal high signal intensity of a disrupted posterior root. $\mathrm{MME}_{\mathrm{US}}$ was compared between MMPRT+ and MMPRT- patients using a nonpaired t-test. Receiver operating characteristic (ROC) curves were used to determine the optimal cut-off $\mathrm{MME}_{\text {US }}$ to predict MMPRT+.

Results: The prevalence of MMPRT+ was 31.3\% (25/80 knees) and 29.1\% (16/55 knees) in the non-ROA and ROA groups. The MME $\mathrm{ES}_{\mathrm{US}}$ of MMPRT + patients were significantly greater than that of MMPRTpatients in both the non-ROA $(5.9 \pm 1.4 \mathrm{~mm}$ vs. $4.4 \pm 1.0 \mathrm{~mm}, \mathrm{P}<0.001)$ and $\mathrm{ROA}$ (7.8 $\pm 1.3 \mathrm{~mm}$ vs. $6.3 \pm 1.3 \mathrm{~mm}, \mathrm{P}<0.001)$ groups. ROC curves demonstrated that 5 -mm and $7-\mathrm{mm} \mathrm{MME}_{\mathrm{US}}$ were the optimal cut-off values in non-ROA (adjusted odds ratio: 6.280; area under the curve [AUC]: 0.809; $P<0.001$ ) and ROA (adjusted odds ratio: 15.003; AUC: 0.797; $P=0.001$ ) groups.

Conclusions: In early non-radiographic KOA stages, a greater $\mathrm{MME}_{\mathrm{US}}$ was associated with a higher MMPRT $_{\text {MRI }}$ prevalence.

\section{Level of evidence: III, Cross-sectional cohort study}

\section{Introduction}

The anterior and posterior meniscal roots are essential structures for anchoring the menisci to the tibial plateau and maintaining the meniscal function of articular cartilage protection. These bony attachments of the menisci allow the circumferential collagen fibers in the meniscal body to disperse axial loads into hoop stresses that redistribute the forces applied to the knee joint during daily activities. ${ }^{1,2}$ Specifically, the posterior root of the medial meniscus is rigidly attached to the tibia, thereby rendering the meniscal body less mobile compared to the other roots. Therefore, the posterior root of the medial meniscus is more vulnerable to not only traumatic damage such as hyperflexion or squatting but also degenerative changes, resulting from a higher incidence of root tears. ${ }^{3,4}$ Medial meniscus posterior root tear (MMPRT), defined as an avulsion injury or radial tear occurring within $10 \mathrm{~mm}$ from a bony attachment, has gained the attention of clinicians and surgeons to deal with knee symptom management. ${ }^{5-7}$ MMPRT substantially disrupts the ability of medial meniscus to anchor the stretching of circumferential collagen 
fibers in a radial direction; the medial meniscus radially extrudes toward the outside of the knee joint during the allocation of axial loading force. ${ }^{3}$ Consequently, MMPRT not only alters loading stress distribution to increase peak contact pressure and decrease contact area in the medial compartment of the tibiofemoral joint ${ }^{3,5,8}$, but also influences joint arthrokinematics by increasing the level of lateral tibial translation and medial compartment excursion. ${ }^{9}$ Intra-articular derangement derived from MMPRT eventually leads to a rapid progression of osteoarthritis $(\mathrm{OA})$ or spontaneous osteonecrosis in the affected knee joint. $5,10-12$

More recently, the concept of early knee OA has drawn the interest of clinicians and researchers in the early diagnosis of knee OA to prevent the progression of OA changes. ${ }^{13,14}$ Accordingly, the early diagnosis of MMPRT prior to definitive knee OA development significantly contributes to the improvement of patient quality of life in terms of locomotive function. Due to the lack of highly sensitive or specific history and physical findings, ${ }^{3}$ the use of magnetic resonance imaging (MRI) has been valuable in MMPRT detection based on the presence of a linear defect around the posterior bony insertion of meniscal roots on axial or coronal view. ${ }^{15-17}$ Moreover, other important findings are observed on sagittal view, such as the "Ghost meniscus sign", which indicates the absence of an identifiable triangular meniscal body as a high signal replacing the normal dark meniscal signal; a normal meniscus is seen on the immediately adjacent images. 3,5,15,17-19 Nevertheless, MRI has limitations such as increased cost and an increased duration of examination. Therefore, the use of a more convenient alternative examination modality to validate the risk of MMPRT would be helpful in the outpatient room, for instance.

Ultrasonography (US) has several advantages over MRI; US provides physicians with an inexpensive, noninvasive, quick, and real-time assessment of the knee joint. ${ }^{20-22}$ Several studies have reported that US demonstrates a relatively high sensitivity and specificity in meniscal pathology detection. ${ }^{20,23,24}$ Limited previous studies have reported that US could indirectly detect greater medial meniscus extrusion (MME) in a knee joint with MMPRT in the biomechanical ${ }^{25}$ or clinical setting ${ }^{26}$, while the available US data on the direct detection of posterior root rupture in the medial joint space are scarce. More recent cohort study reported that a knee joint with a greater MME measured by US (MME $\mathrm{US}_{\mathrm{S}}$ ) had a higher risk of knee OA development or aggravation regardless of MMPRT occurrence. ${ }^{22}$ Thereafter, $\mathrm{MME}_{\mathrm{US}}$ has the potential to indirectly reveal the presence of MMPRT in patients having knee symptoms with a predisposition for knee $\mathrm{OA}$ development or aggravation. Unfortunately, it is difficult to validate the sensitivity of $\mathrm{MME}_{\mathrm{US}}$ to indicate the presence of MMPRT due to a lack of previous data. Thus, we aimed to cross-sectionally evaluate how the value of $M_{M} E_{U S}$ can indicate the presence of MMPRT on MRI (MMPRT ${ }_{M R I}$ ), dividing the patients who have medial knee joint pain into non-radiographic knee OA and radiographic knee OA. We hypothesized that the greater value of non-weight bearing $\mathrm{MME}_{U S}$ with patients in the supine position would be associated with a higher prevalence of MMPRT $_{\text {MRI}}$.

\section{Methods}

\section{Study participants}


The current study was approved by the ethical committee of Hirosaki Memorial Hospital (IRB No. 202013). All participants were informed of the aims and risks of the current study and agreed to participate in this study. All methods were performed in accordance with the relevant guidelines and regulations (Declaration of Helsinki). Study participants visited our hospital from December 2019 to December 2020 presenting with medial knee joint pain during daily activities such as walking, climbing and descending stairs, and sitting and standing. During this period, 186 patients visited our outpatient clinic. We excluded the patients without MRI or US data, and those with a history of knee trauma or surgery. Finally, we recruited 127 patients (135 knees) for the current study.

Weight bearing anteroposterior knee radiographs were acquired with film-focus distance of $100 \mathrm{~cm}, 68 \mathrm{kV}$, and 20 mAs. Plain radiographs of the affected knee were taken in an orthostatic position with the knee semi-flexed, corresponding to Rosenberg view. According to the Kellgren-Lawrence grade $(\mathrm{KLG})^{27}$, single orthopaedic surgeon (DC, 12-year experience) assigned participants to the non-radiographic knee OA (Non-ROA: KLG 0-1) or radiographic knee OA (ROA: KLG 2-4) group. The intra-rater reliability (k) for determining KLG was 0.821 for the right knee and 0.805 for the left knee. ${ }^{28}$

\section{Ultrasonographic measurement of MME}

All participants underwent measurement of non-weight bearing $\mathrm{MME}_{\mathrm{US}}$ in the supine position. The ultrasound probe (12 MHz, ARIETTA Prologue, Hitachi Aloka Medical, Tokyo, Japan) was placed at the center of the medial knee joint space, with the knee joint fully extended and in neutral rotation. MME was measured on ultrasonographic image where the medial meniscus was displayed with a hypoechoic band of the medial collateral ligament ${ }^{29}$ and the downslope of the medial femoral epicondyle. (Fig. 1) First, a line was drawn connecting the cortex of both the femur and tibia (Line A); thereafter, a line perpendicular to Line A was drawn from the bottom of Line A to the most medially extruded part of the medial meniscus. (Line B, Fig. 1) Finally, the length of Line B was measured as the $M_{M} E_{U s}$. Regarding the technique used to draw Line A, we ignored the existence of osteophytes and drew Line A through the osteophyte bases to avoid the bony interference of osteophytes for Line B. Additionally, on the femoral side of Line $A$, this line should trace the femoral cortex at the bottom of the medial femoral epicondyle. (Fig. 1) In the outpatient room, single orthopaedic surgeon (DC) acquired US image and measured MME before the patients underwent the plain radiographs of affected knee. Regarding the intra-rater reliability of DC, intraclass correlation coefficient [ICC] (1.1) was $0.977(95 \% \mathrm{Cl}: 0.942-0.991)^{22}$

\section{MRI evaluation of MMPRT}

All participants underwent 1.5-T MRI for the affected knee joint (Optima MR360, GE Healthcare). The knee joint was kept immobilized by customized flame during examination with $10^{\circ}$ knee flexion and externally rotated at $10-15^{\circ}$. A T2*-weighted image sequence was used to evaluate MMPRT. (TR/TE[ms]: 600700/8.6, field of view: 180×180 mm, Matrix 256×224, Layer thickness: 4.0 mm, Gap: $1.0 \mathrm{~mm}$ ) Based on the sagittal images, the presence of MMPRT was defined using the ghost meniscus sign, which indicates the absence of an identifiable triangular meniscal body as a high signal replacing the normal dark meniscal signal seen on the immediately adjacent images. 5,17,19 (Fig. 2a) Furthermore, on coronal MRI, 
cleft/truncation sign (vertical linear defect) was defined as the other reference of MMPRT. (Fig. 2b) If the current subjects had both ghost meniscus and cleft/truncation signs, they were defined as having MMPRT. Regarding the diagnostic performance of MMPRT, both ghost meniscus (96.7-100\%) and cleft/truncation sign (90-100\%) have been reported to demonstrate a high sensitivity. ${ }^{16,17}$ Radiologist in our hospital finally diagnosed the presence of MMPRT $_{{ }_{M R l}}$, blinded to the clinical information of current subjects.

\section{Statistical analysis}

All statistical analyses were conducted using SPSS version 24 (SPSS Inc., Chicago, IL, USA). Continuous variables of demographic data and $\mathrm{MME}_{U S}$ between patients with and without the MMPRT MRI $_{\text {were }}$ compared by non-paired t-test. A receiver operating characteristic (ROC) curve was drawn to determine

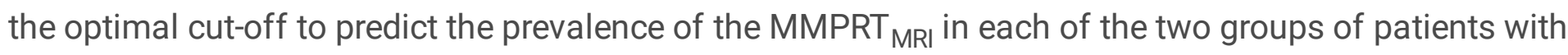
knee OA. Using the optimal cut-off of $\mathrm{MME}_{U S}$, a logistic regression analysis was conducted with the prevalence of the MMPRT ${ }_{M R I}$ as the dependent variable, and with the optimal cut-off of $M_{M M}$ as the independent variable, adjusted for age, sex, and body mass index. A P-value $<0.05$ was considered statistically significant.

\section{Results}

The prevalence of positive MMPRT (MMPRT+) was 31.3\% (25/80 knees) and $29.1 \%$ (16/55 knees) in the non-ROA and ROA groups, respectively. The prevalence of MMPRT + was not significantly different between the groups. In the non-ROA group, the mean age of patients in the MMPRT + group was significantly higher than that of patients in the negative MMPRT (MMPRT-) group. (Table 1) MME $\mathrm{US}_{\text {in }}$ MMPRT + patients were significantly greater than that in MMPRT- patients in both the non-ROA (5.9 \pm 1.4 $\mathrm{mm}$ vs. $4.4 \pm 1.0 \mathrm{~mm}, \mathrm{P}<0.001)$ and the ROA groups $(7.8 \pm 1.3 \mathrm{~mm}$ vs. $6.3 \pm 1.3 \mathrm{~mm}, \mathrm{P}<0.001$, Table 1$)$. 
Table 1

Current participant demographic characteristics

\begin{tabular}{|c|c|c|c|c|c|c|c|c|}
\hline \multirow[b]{2}{*}{ Non-ROA } & \multirow[b]{2}{*}{ Age, year } & \multicolumn{3}{|c|}{ MMPRT- $(\mathrm{N}=55)$} & \multicolumn{3}{|c|}{ MMPRT+ $(\mathrm{N}=25)$} & \multirow{2}{*}{$\begin{array}{l}\text { P-value } \\
0.004\end{array}$} \\
\hline & & 61.3 & \pm & 9.3 & 67.6 & \pm & $7.4^{*}$ & \\
\hline & $\operatorname{Sex}(F / M)$ & 29 & / & 26 & 18 & / & 7 & 0.105 \\
\hline & $\mathrm{BMI}, \mathrm{kg} / \mathrm{m}^{2}$ & 24.6 & \pm & 2.9 & 25.3 & \pm & 3.3 & 0.332 \\
\hline & MME, mm & 4.4 & \pm & 1.0 & 5.9 & \pm & $1.4^{*}$ & $<0.001$ \\
\hline & & \multicolumn{3}{|c|}{ MMPRT- $(\mathrm{N}=39)$} & \multicolumn{3}{|c|}{ MMPRT+ $(\mathrm{N}=16)$} & P-value \\
\hline \multirow[t]{4}{*}{ ROA } & Age, year & 66.1 & \pm & 8.0 & 66.1 & \pm & 7.0 & 0.992 \\
\hline & $\operatorname{Sex}(F / M)$ & 31 & / & 8 & 12 & / & 4 & 0.714 \\
\hline & $\mathrm{BMI}, \mathrm{kg} / \mathrm{m}^{2}$ & 26.5 & \pm & 3.8 & 26.0 & \pm & 3.1 & 0.644 \\
\hline & $\mathrm{MME}, \mathrm{mm}$ & 6.3 & \pm & 1.3 & 7.8 & \pm & $1.3^{*}$ & $<0.001$ \\
\hline
\end{tabular}

The ROC curve demonstrated that the MME $\mathrm{MS}_{\text {v }}$ value could significantly predict the prevalence of MMPRT + in both the non-ROA (AUC: 0.809, $\mathrm{P}<0.001$ ) and the ROA (AUC: 0.797, $\mathrm{P}=0.001$ ) group. Based on the ROC curve, the optimal cut-offs of $M_{M E_{U S}}$ in the non-ROA and ROA groups were $5 \mathrm{~mm}$ (Sensitivity: $76.0 \%$ and Specificity: $73.6 \%$ ) and $7 \mathrm{~mm}$ (Sensitivity: $81.3 \%$ and Specificity: $74.4 \%$ ), respectively. (Fig. 3)

Multiple logistic regression analysis showed that a 5-mm MME US was significantly associated with the prevalence of MMPRT + in the non-ROA group (adjusted odds ratio: $6.280 ; 95 \% \mathrm{Cl}$ : 1.854 to $21.277 ; \mathrm{P}=$ 0.003). Similarly, a 7-mm MME $\mathrm{mS}_{\mathrm{S}}$ was associated with the prevalence of MMPRT + in the ROA group (adjusted odds ratio: $15.003 ; 95 \% \mathrm{Cl}: 3.923$ to $69.205 ; \mathrm{P}=0.001$, Table 2). 
Table 2

Logistic regression analysis to elucidate the relationship between the cut-off value of medial meniscus extrusion and the prevalence of the ghost meniscus sign.

\begin{tabular}{|c|c|c|c|c|c|c|c|c|}
\hline Cut-off value & & Model & B & P-value & OR & $95 \% \mathrm{Cl}$ & & \\
\hline \multirow[t]{2}{*}{ MME 5 mm } & Non-ROA & Crude & 2.277 & $<0.001$ & 9.750 & 3.119 & - & 30.477 \\
\hline & & Adjusted & 1.837 & 0.003 & 6.280 & 1.854 & - & 21.277 \\
\hline \multirow[t]{4}{*}{ MME 6 mm } & Non-ROA & Crude & 1.897 & 0.002 & 6.667 & 1.971 & - & 22.553 \\
\hline & & Adjusted & 1.542 & 0.024 & 4.674 & 1.222 & - & 17.877 \\
\hline & $\mathrm{ROA}$ & Crude & 2.345 & 0.030 & 10.435 & 1.249 & - & 87.144 \\
\hline & & Adjusted & 2.678 & 0.018 & 14.554 & 1.588 & - & 133.344 \\
\hline \multirow[t]{2}{*}{ MME $7 \mathrm{~mm}$} & ROA & Crude & 2.531 & 0.001 & 12.567 & 2.958 & - & 53.390 \\
\hline & & Adjusted & 2.708 & 0.001 & 15.003 & 3.253 & - & 69.205 \\
\hline \multicolumn{9}{|c|}{$\begin{array}{l}\text { B: regression coefficient; MME: medial meniscus extrusion; OR: odds ratio; ROA: radiographic knee } \\
\text { osteoarthritis (Kellgren-Lawrence grade } \geq 2 \text { ), } 95 \% \mathrm{Cl} \text { : } 95 \% \text { confidence interval. The MME cut-off values } \\
\text { of } 5 \mathrm{~mm} \text { in ROA and } 7 \mathrm{~mm} \text { in non-ROA group are not significantly associated with the prevalence of } \\
\text { ghost meniscus sign. }\end{array}$} \\
\hline
\end{tabular}

\section{Discussion}

The most important finding of the current study is that a greater $\mathrm{MME}_{\mathrm{US}}$ was associated with a higher prevalence of MMPRT ${ }_{M R I}$ based on a positive finding of the ghost meniscus and creft/truncation signs, which corresponds to the rupture of the posterior root of the medial meniscus on sagittal and coronal T2*-weighted MRI. Notably, the current finding was consistently significant in patients with medial knee pain both with and without definitive radiographic OA changes defined by the KLG. Moreover, the cut-off value of $M_{M E_{U S}}$ differed according to the stage of radiographic knee OA.

The prevalence of MMPRT in the current study was 31.3\% (25/80 knees) and 29.1\% (16/55 knees) in the non-ROA and ROA groups. This prevalence of MMPRT was higher than the previous epidemiological data, ranged from 10.1 to $27.8 \% .{ }^{30-32}$ Interestingly, Bin et al. ${ }^{30}$ and Hwang et al. ${ }^{32}$ from Korea reported relatively similar prevalence of MMPRT with the current study. Asian people are more likely to experience MMPRT due to their lifestyle of frequent squatting and sitting on the floor with the legs deeply folded. ${ }^{5} \mathrm{~A}$ greater $\mathrm{MME}_{U S}$ was consistently associated with a higher prevalence of MMPRT ${ }_{M R}$, thereby verifying our study hypothesis. In accordance with the significant loss of meniscal function resulting from MMPRT, some previous cohort studies reported that patients with medial meniscus posterior root injury on $\mathrm{MRI}^{33,34}$ demonstrate a greater MME on the corresponding MRI. In line with these previous cohort study findings, our study findings showed that the use of US is compatible with that of MRI in terms of 
measuring MME for MMPRT prevalence determination. Furthermore, US is likely to be clinically relevant for the easily validation of the risk of MMPRT in patients with medial knee joint pain in the outpatient clinic.

However, there is insufficient evidence to connect the relationship between $\mathrm{MME}_{\mathrm{US}}$ and the prevalence of MMPRT at this point. A cadaveric biomechanical study reported that the resection of the posterior root of the lateral meniscus affected the degree of $\mathrm{MME}_{\mathrm{US}} \cdot{ }^{25}$ For knees with a total resection of the posterior root, a greater $\mathrm{MME}_{\mathrm{US}}$ was observed in comparison to that of knees with a partial resection. Specifically, when an axial load was applied, $M_{M} E_{U S}$ was significantly greater than when a non-axial load was applied; the latter was simulated as non-weight bearing condition. ${ }^{25}$ Karpinski et al. conducted a study with a similar design to that of the current study to elucidate the relationship between the prevalence of MMPRT $_{M R I}$ and the values of MME measured by both US and MRI. ${ }^{26}$ They observed a greater MME value in participants who had MMPRT in the knee OA population with a relatively early stage of KLG $0-2$. This study ${ }^{26}$ also evaluated the alterations between the weight and non-weight bearing conditions of $\mathrm{MME}_{\mathrm{US}}$. Interestingly, the results of Karpinski et al. conflicted with those of Rowland et al. ${ }^{25}$ wherein weight bearing condition did not change the value of $\mathrm{MME}_{\mathrm{US}}{ }^{26}$ Based on these limited US data, there remains a controversy with regard to the relationship between the value of $M_{M M}$ and the prevalence of MMPRT. The biomechanical effect of MMPRT on MME $\mathrm{MS}_{\text {S }}$ would change in accordance with the severity of cartilage degeneration, ${ }^{35,36}$ lateral or medial meniscus involvement, and weight or non-weight bearing condition. Moreover, to improve inter-rater reliability, future studies should be conducted to determine a consistent method of $\mathrm{MME}_{\mathrm{US}}$ measurements.

The most important limitation of previous US studies ${ }^{25,26}$ is the small sample size; the reliability of $M_{M} E_{U S}$ was not high enough to determine the prevalence of MMRPT. Thus, the lack of evidence makes it difficult for musculoskeletal healthcare providers to consider the values of $M_{M} E_{U S}$ that validate the prevalence of MMPRT at various stages of knee OA. Based on our study data, with a relatively large sample size, we can progress in the further discussion of applying the optimal cut-off of $M_{M E_{U S}}$ to determine the prevalence of MMPRT. In the current non-radiographic OA population with KLG 0-1, a 5mm MME $E_{U S}$ is the optimal cut-off to detect MMPRT on T2*-weighted sagittal MRI. In contrast, a 7-mm $M \mathrm{ME}_{U S}$ cut-off is optimal for detecting MMPRT in the definitive radiographic OA population with KLG $\geq 2$. Compared to the findings of Karpinski et al., the current cut-off values are greater; notably, the upper error bar of the supine position $\mathrm{MME}_{\mathrm{US}}$ was between $5 \mathrm{~mm}$ and $6 \mathrm{~mm}$ in the study by Karpinski et al. ${ }^{26}$ In other words, further discussion would be needed to conclude which $\mathrm{MME}_{\mathrm{US}}$ value is best to detect the prevalence of MMPRT, and future large-sample studies will shed light on the detailed mechanism of the effect of meniscus root rupture on the deterioration of meniscal function.

Our study had several limitations. First, we could not validate whether the current study participants had grossly visible MMPRT using arthroscopy. Second, the US evaluation was performed only in the supine position with a non-weight bearing condition. Therefore, we did not discuss how the weight bearing 
condition affects $M_{M} E_{U S}$ based on the current data. Third, the sample size of the advanced knee $O A$ stage ( $K L G 3-4, N=20$ knees) was relatively small. Finally, the design of the current study was crosssectional, and therefore we could not establish a cause-effect relationship between $\mathrm{MME}_{\mathrm{US}}$ value and MMPRT development or aggravation. Despite these limitations, the current study emphasizes the clinical relevance of $\mathrm{MME}_{U S}$ in detecting the prevalence of MMPRT by healthcare providers. The use of US can potentially aid in the evaluation of posterior root meniscus rupture in an outpatient room.

\section{Conclusion}

The current cross-sectional study elucidated the association between $\mathrm{MME}_{\mathrm{US}}$ and the prevalence of MMPRT. A greater MME $\mathrm{MS}_{\text {S }}$ was associated with a higher prevalence of MMPRT on T2*-weighted MRI. In particular, $\mathrm{MME}_{\mathrm{US}} \geq 5 \mathrm{~mm}$ can be a risk factor for MMPRT in patients with medial knee joint pain and non-radiographic knee $\mathrm{OA}$.

\section{Declarations}

\section{Acknowledgements:}

We would like to thank Editage (https://www.editage.jp) for English language editing.

\section{Authors' Contribution:}

D.C. wrote the main manuscript and designed the current study.

T.S. approved the current study design and contribute to the data collection.

Y.I. revised the main manuscript and approved the current study design.

\section{Disclosures:}

There is nothing to declare.

\section{References}

1. Chung, K. S., Ha, J. K., Ra, H. J., Nam, G. W. \& Kim, J. G. Pullout Fixation of Posterior Medial Meniscus Root Tears: Correlation Between Meniscus Extrusion and Midterm Clinical Results. Am J Sports Medicine, 45, 42-49 (2016).

2. LaPrade, R. F., Matheny, L. M., Moulton, S. G., James, E. W. \& Dean, C. S. Posterior Meniscal Root Repairs: Outcomes of an Anatomic Transtibial Pull-Out Technique. Am J Sports Medicine, 45, 884891 (2017). 
3. Bhatia, S., LaPrade, C. M., Ellman, M. B. \& LaPrade, R. F. Meniscal Root Tears. Am J Sports Medicine, 42, 3016-3030 (2014).

4. Kodama, Y. et al. Transtibial pullout repair of medial meniscus posterior root tears: effects on the meniscus healing score and ICRS grade among patients with mild osteoarthritis of the knee. Knee Surg Sports Traumatol Arthrosc Online ahead of print(2020) doi:10.1007/s00167-020-06332-7.

5. Petersen, W. et al. Posterior root tear of the medial and lateral meniscus. Arch Orthop Trauma Surg, 134, 237-255 (2013).

6. Chung, K. S., Ha, J. K., Ra, H. J. \& Kim, J. G. A meta-analysis of clinical and radiographic outcomes of posterior horn medial meniscus root repairs. Knee Surg Sports Traumatol Arthrosc, 24, 1455-1468 (2015).

7. Chahla, J. \& LaPrade, R. F. Meniscal Root Tears. Arthroscopy, 35, 1304-1305 (2019).

8. Allaire, R., Muriuki, M., Gilbertson, L. \& Harner, C. D. Biomechanical Consequences of a Tear of the Posterior Root of the Medial Meniscus. J Bone Joint Surg, 90, 1922-1931 (2008).

9. Marsh, C. A., Martin, D. E., Harner, C. D. \& Tashman, S. Effect of Posterior Horn Medial Meniscus Root Tear on In Vivo Knee Kinematics. Orthop J Sports Medicine, 2, 2325967114541220 (2014).

10. Yamagami, R., Taketomi, S., Inui, H., Tahara, K. \& Tanaka, S. The role of medial meniscus posterior root tear and proximal tibial morphology in the development of spontaneous osteonecrosis and osteoarthritis of the knee. Knee, 24, 390-395 (2017).

11. Park, J. Y., Kim, B. H., Ro, D. H., Lee, M. C. \& Han, H. S. Characteristic location and rapid progression of medial femoral condylar chondral lesions accompanying medial meniscus posterior root tear. Knee, 26, 673-678 (2019).

12. Kamatsuki, Y. et al. Placement of an anatomic tibial tunnel significantly improves the medial meniscus posterior extrusion at $90^{\circ}$ of knee flexion following medial meniscus posterior root pullout repair. Knee Surg Sports Traumatol Arthrosc Online ahead of print(2020) doi:10.1007/s00167-02006070-w.

13. Sasaki, E. et al. Early knee osteoarthritis prevalence is highest among middle-aged adult females with obesity based on new set of diagnostic criteria from a large sample cohort study in the Japanese general population. Knee Surg Sports Traumatol Arthrosc, 28, 984-994 (2019).

14. Luyten, F. P. et al. Toward classification criteria for early osteoarthritis of the knee. Semin Arthritis Rheum, 47, 457-463 (2018).

15. Kim, S. B. et al. Medial Meniscus Root Tear Refixation: Comparison of Clinical, Radiologic, and Arthroscopic Findings With Medial Meniscectomy., 27, 346-354 (2011).

16. Lee, Y. G. et al. Magnetic Resonance Imaging Findings of Surgically Proven Medial Meniscus Root Tear. J Comput Assist Tomogr, 32, 452-457 (2008).

17. Choi, S. H., Bae, S., Ji, S. K. \& Chang, M. J. The MRI findings of meniscal root tear of the medial meniscus: emphasis on coronal, sagittal and axial images. Knee Surg Sports Traumatol Arthrosc, 20, 2098-2103 (2011). 
18. Lerer, D. B., Umans, H. R., Hu, M. X. \& Jones, M. H. The role of meniscal root pathology and radial meniscal tear in medial meniscal extrusion. Skeletal Radiol, 33, 569-574 (2004).

19. Harper, K. W., Helms, C. A., Lambert, H. S. \& Higgins, L. D. Radial Meniscal Tears: Significance, Incidence, and MR Appearance. AJR Am J Roentgenol, 185, 1429-1434 (2005).

20. Friedman, L., Finlay, K. \& Jurriaans, E. Ultrasound of the knee. Skeletal Radiol, 30, 361-377 (2001).

21. Razek, A. A. K. A., Fouda, N. S., Elmetwaley, N. \& Elbogdady, E. Sonography of the knee joint. J Ultrasound, 12, 53-60 (2009).

22. Chiba, D. et al. US detection of medial meniscus extrusion can predict the risk of developing radiographic knee osteoarthritis: a 5-year cohort study. Eur Radiol, 30, 3996-4004 (2020).

23. Park, G. Y., Kim, J. M., Lee, S. M. \& Lee, M. Y. The Value of Ultrasonography in the Detection of Meniscal Tears Diagnosed by Magnetic Resonance Imaging. Am J Phys Med Rehab, 87, 14-20 (2008).

24. Wareluk, P. \& Szopinski, K. T. Value of modern sonography in the assessment of meniscal lesions. Eur J Radiol, 81, 2366-2369 (2012).

25. Rowland, G., Mar, D., Mclff, T. \& Nelson, J. Evaluation of meniscal extrusion with posterior root disruption and repair using ultrasound. Knee, 23, 627-630 (2016).

26. Karpinski, K. et al. No dynamic extrusion of the medial meniscus in ultrasound examination in patients with confirmed root tear lesion. Knee Surg Sports Traumatol Arthrosc, 27, 3311-3317 (2019).

27. Kellgren, J. H. \& Lawrence, J. S. Radiological Assessment of Osteo-Arthrosis. Ann Rheum Dis, 16, 494-502 (1957).

28. Chiba, D. et al. Low prevalence of knee chondrocalcinosis and its catabolic association with serum matrix metalloproteinase 3: A rural Japanese population study. Int J Rheum Dis, 21, 2011-2018 (2018).

29. Kawaguchi, K., Enokida, M., Otsuki, R. \& Teshima, R. Ultrasonographic evaluation of medial radial displacement of the medial meniscus in knee osteoarthritis. Arthritis Rheum, 64, 173-180 (2012).

30. Bin, S. I., Kim, J. M. \& Shin, S. J. Radial tears of the posterior horn of the medial meniscus., 20, 373378 (2004).

31. Ozkoc, G. et al. Radial tears in the root of the posterior horn of the medial meniscus. Knee Surg Sports Traumatol Arthrosc, 16, 849-854 (2008).

32. Hwang, B. Y. et al. Risk Factors for Medial Meniscus Posterior Root Tear. Am J Sports Medicine, 40, 1606-1610 (2012).

33. Choi, C. J., Choi, Y. J., Lee, J. J. \& Choi, C. H. Magnetic Resonance Imaging Evidence of Meniscal Extrusion in Medial Meniscus Posterior Root Tear., 26, 1602-1606 (2010).

34. Magee, T. MR findings of meniscal extrusion correlated with arthroscopy. J Magn Reson Imaging, 28, 466-470 (2008). 
35. Svensson, F. et al. Scrutinizing the cut-off for "pathological" meniscal body extrusion on knee MRI. Eur Radiol, 29, 2616-2623 (2019).

36. Aoki, H. et al. Relationship between medial meniscus extrusion and cartilage measurements in the knee by fully automatic three-dimensional MRI analysis. BMC Musculoskelet Disord, 21, 742 (2020).

\section{Figures}

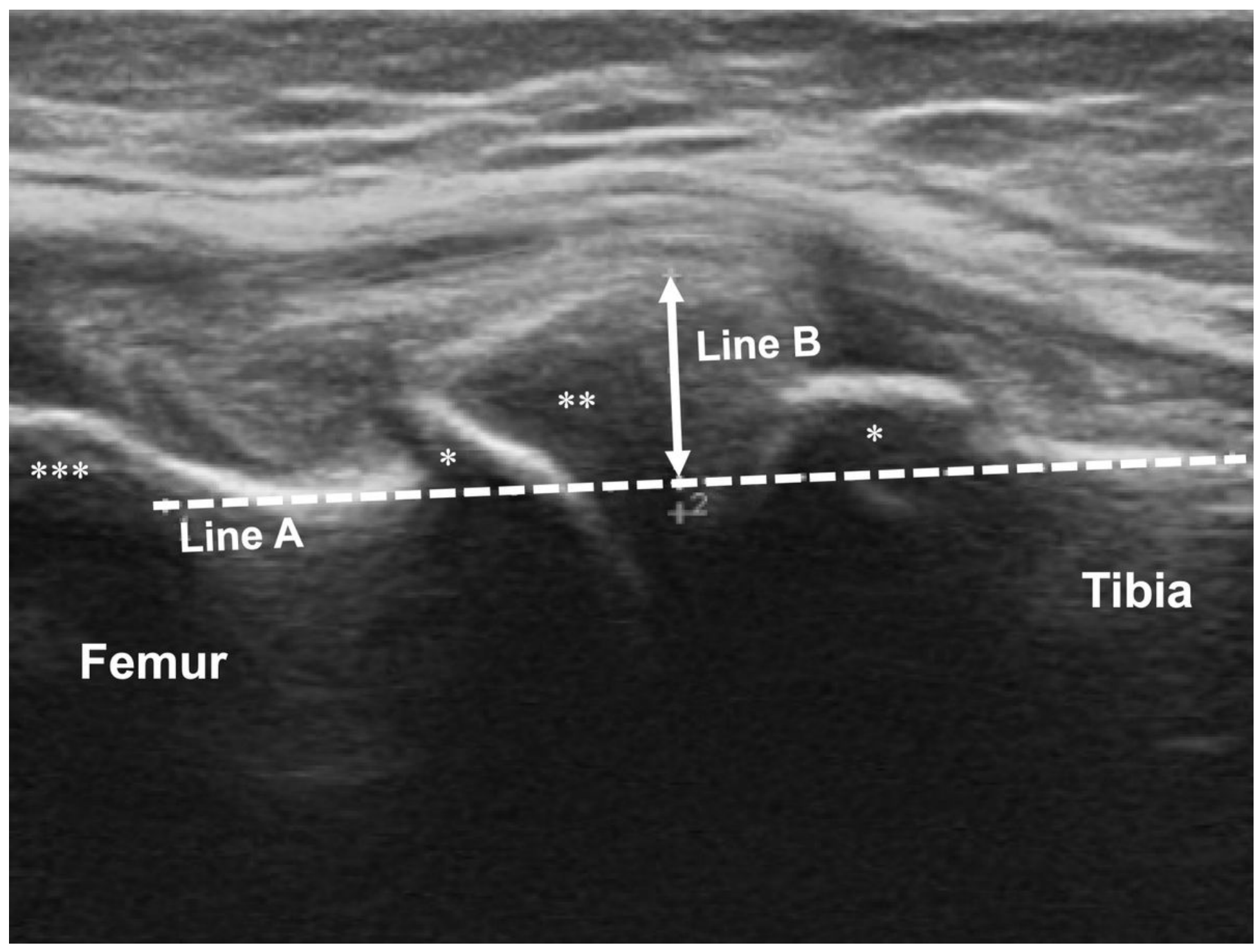

\section{Figure 1}

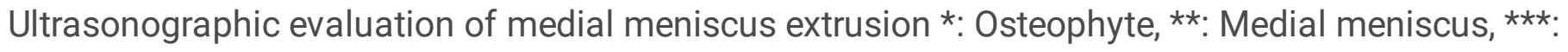
Medial femoral epicondyle. Line A was drawn to connect the cortex of both the femur and tibia, thereby tracing the femoral cortex at the bottom of the medial femoral epicondyle. Line B was drawn perpendicularly from the bottom of Line A to the most medially extruded part of the medial meniscus. Line A was drawn through the osteophyte bases to avoid the bony interference of osteophytes throughout the length of Line B. Finally, the length of Line B $(\mathrm{mm})$ was measured as the medial meniscus extrusion. 


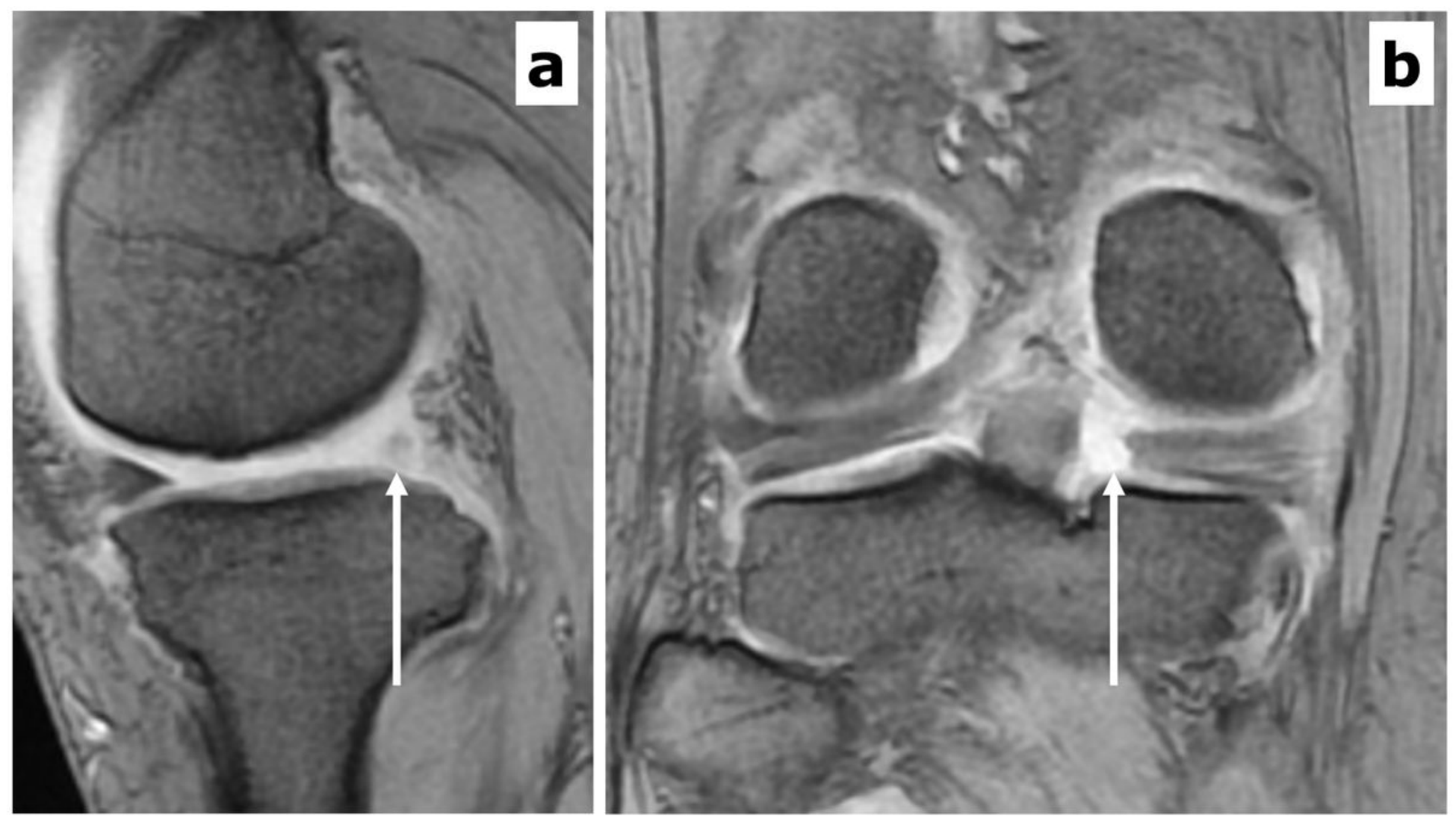

\section{Figure 2}

Ghost meniscus sign on a T2*-weighted sagittal image a: Sagittal view showing ghost meniscus sign b: Coronal view showing a defect of the medial meniscus posterior root. An abnormally high signal, indicating the absence of an identifiable meniscal body, is detected in place of the normal dark meniscal signal at the posterior horn of the medial meniscus. (Arrow) 

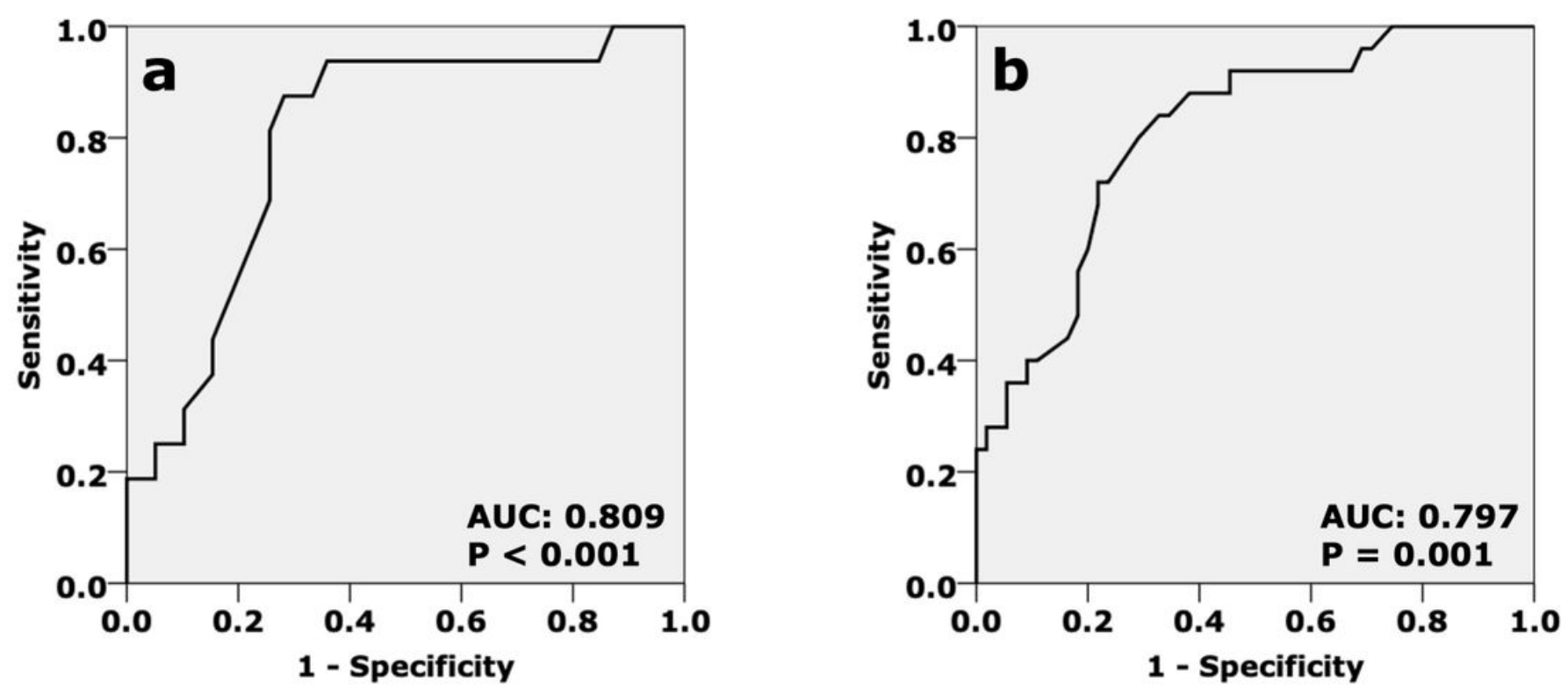

Figure 3

Receiver operating characteristic curve to predict the ghost meniscus sign on a T2*-weighted sagittal image a: Non-ROA group; b: ROA group; AUC: area under curve; ROA: radiographic knee osteoarthritis (Kellgren-Lawrence grade $\geq 2$ ). 